\title{
Anterolateral Approach with Endoscopic Assistance for Resection of a Craniovertebral Junction Chordoma
}

\author{
Pierre-Olivier Champagne ${ }^{1}$ Eduard H. Voormolen ${ }^{1} \quad$ Sébastien Froelich $^{1}$

\footnotetext{
${ }^{1}$ Department of Neurosurgery, Lariboisiere Hospital, University of Paris Diderot, Paris, France
} \\ J Neurol Surg B 2019;80(suppl S4):S375-S377. \\ Address for correspondence Pierre-Olivier Champagne, MD, \\ Department of Neurosurgery, Lariboisière Hospital, University of Paris \\ Diderot, 2 rue Ambroise Pare, Paris, 75010, France \\ (e-mail: pierreolivier.champagne@aphp.fr).
}

\begin{abstract}
Keywords

- anterolateral approach

- chordoma

- craniovertebral junction

- endoscopic-assisted microsurgery

- operative video

Craniovertebral junction chordomas can represent a surgical challenge, especially when intradural extension and contact with neurovascular structures is involved. To date, there is still controversy on the choice of optimal surgical corridor for such lesions, with endoscopic endonasal, far lateral and anterolateral corridors being those most commonly used. In this operative video, we present a case of craniovertebral junction chordoma with significant bone destruction and intradural extension (-Fig. 1). The tumor was safely removed using an anterolateral approach with transposition of the vertebral artery to gain access to the lateral craniovertebral junction. The endoscope is demonstrated to help with intradural tumor removal, providing a wide angle of view through a relatively narrow dural opening (-Fig. 2). In this video, nuances of the anterolateral approach, chordoma removal strategies and closure technique are presented in detail. A gross total resection was achieved, and the postoperative course was uneventful. In summary, the anterolateral approach with endoscopic assistance can offer a safe and effective corridor for the surgical management of craniovertebral junction chordomas.

The link to the video can be found at: https://youtu.be/pCOYxKgNoMY.
\end{abstract}

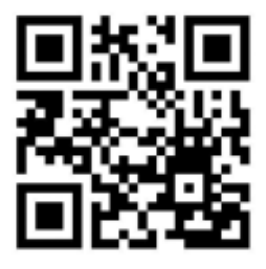

received

February 28, 2019 accepted

August 25, 2019

published online

October 28, 2019 www.thieme.com/skullbasevideos

www.thieme.com/jnlsbvideos

DOI https://doi.org/

10.1055/s-0039-1698820.

ISSN 2193-6331.
๑) 2019 Georg Thieme Verlag KG
Stuttgart · New York

License terms

(c) (1) $\ominus$ (\$) 

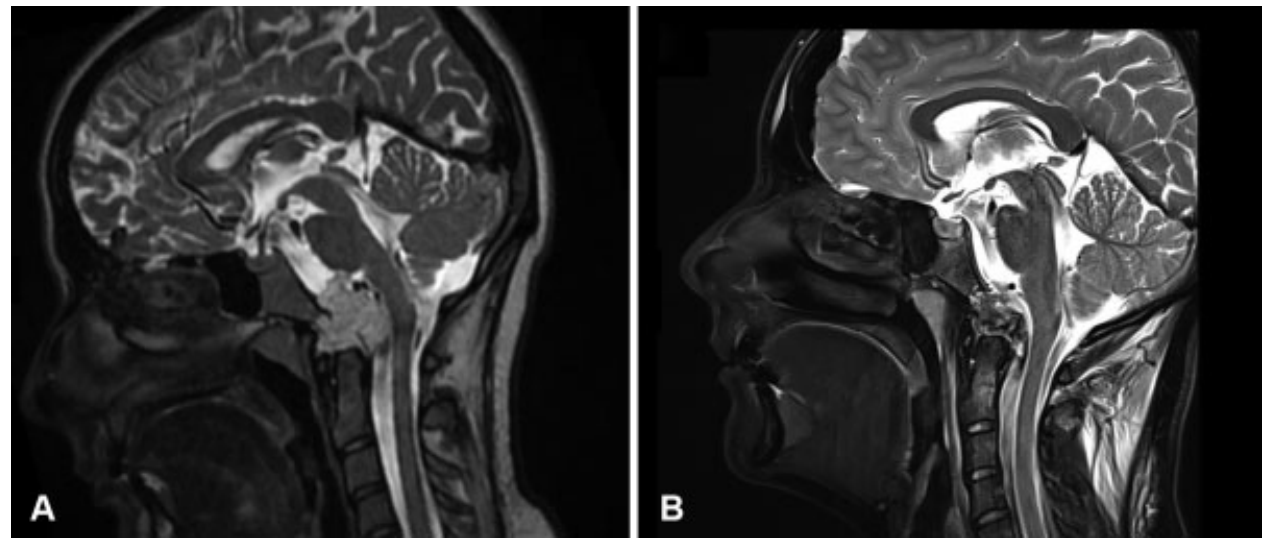

Fig. 1 Preoperative and postoperative magnetic resonance imaging (MRI). (A) Preoperative sagittal T2-weighted MRI showing a hyperintense lesion (chordoma) centered on the anterior craniovertebral junction with intradural extension and compression of neurovascular structures. (B) Postoperative sagittal T2-weighted MRI showing gross total of the tumor.
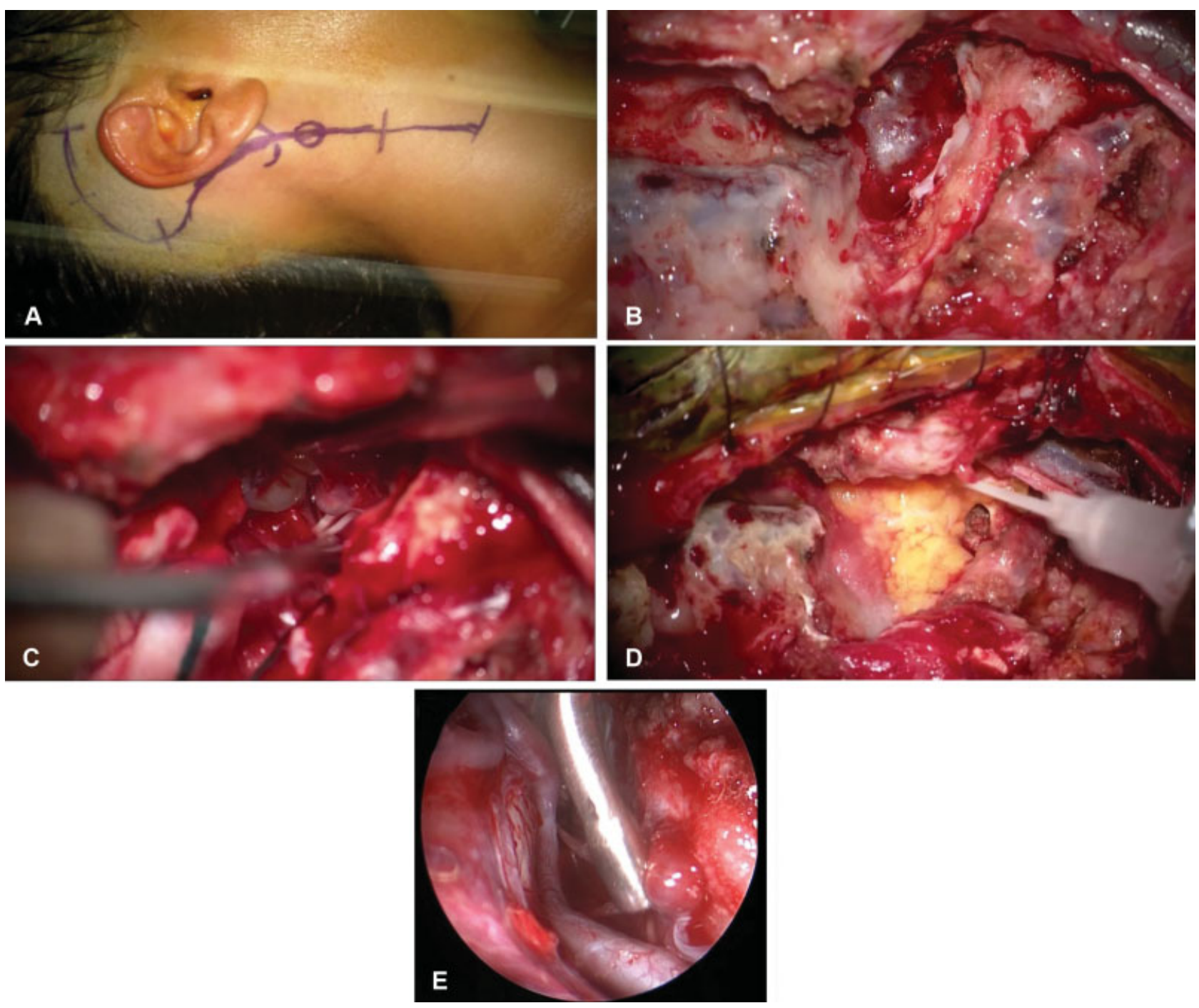

Fig. 2 Surgical steps of surgery. (A) Incision along the anterior border of the sternocleido mastoid muscle and passing above the mastoid process. (B) View of the tumor capsule prior to removal and after vertebral transposition and drilling of the occipital condyle and mastoid tip. (C) Intradural tumor dissection from the nerves of the contralateral jugular foramen. (D) Closure of the dural defect using free fat graft. (E) Endoscopic intradural exploration showing a tumor remnant on the contralateral jugular foramen. 


\section{Publication Comments}

This is an excellent video of a relatively uncommon approach to the craniovertebral junction. This had been initially described by Bernard George and the authors demonstrate a mastery of it. As with most approaches to chordomas, there are certain blind spots, such as the ipsilateral side, superiorly above the jugular foramen, and across the midline onto the opposite side. Since the goal is to drill as much of the surrounding bone as possible, these blind spots are a limitation. Nevertheless it is an effective approach in selected cases that the skull base surgeon should be familiar with. I am not sure how the cement implant at the end of the operation is expected to provide stability.

Chandra Sen, MD New York University School of Medicine New York, NY
The authors present an excellent video of an upper cervical anterior lateral and transmastoid suboccipital approach for resection of an intra- and extradural chordoma of the inferior clivus and upper cervical region ventral to the cervical medullary junction. The surgical technique is excellent and very well displayed in the edited and narrated video along with appropriate annotations of the important anatomy. This video demonstrates masterful technique.

Michael Chicoine, MD

Washington University

St. Louis, MO

Conflict of Interest

None declared. 